University of Wollongong

Research Online

Faculty of Engineering and Information

Faculty of Engineering and Information

Sciences - Papers: Part A

Sciences

$1-1-2012$

Parameter estimation of a regime-switching model using an inverse stieltjes moment approach

$\mathrm{XX \textrm {i }}$

University of Western Ontario

Marianito R. Rodrigo

University of Wollongong, marianit@uow.edu.au

Rogemar S. Mamon

University of Western Ontario

Follow this and additional works at: https://ro.uow.edu.au/eispapers

Part of the Engineering Commons, and the Science and Technology Studies Commons

Research Online is the open access institutional repository for the University of Wollongong. For further information contact the UOW Library: research-pubs@uow.edu.au 


\title{
Parameter estimation of a regime-switching model using an inverse stieltjes moment approach
}

\author{
Abstract \\ We address the problem of recovering the time-dependent parameters of the Black-Scholes option pricing \\ model when the underlying stock price dynamics are modelled by a finite-state, continuous-time Markov \\ chain. The coupled system of Dupire-type partial differential equations is derived and formulated as an \\ inverse Stieltjes moment problem. We provide numerical illustration on how to apply our method to \\ simulated financial data. The accuracy of the model parameter estimation is examined and sensitivity \\ analyses are included to study the behaviour of the estimated results when model parameters are varied.

\section{Keywords} \\ estimation, inverse, regime, parameter, approach, stieltjes, moment, switching, model \\ Disciplines \\ Engineering | Science and Technology Studies

\section{Publication Details} \\ Xi, X., Rodrigo, M. R. \& Mamon, R. S. (2012). Parameter estimation of a regime-switching model using an \\ inverse stieltjes moment approach. In S. N. Cohen, D. Madan, T. Kuen. Siu \& H. Yang (Eds.), Stochastic \\ processes, finance and control : a festschrift in honor of Robert J. Elliott (pp. 549-569). Singapore: World \\ scientific.
}




\section{Chapter 22}

Parameter estimation of a regime-switching model using an inverse Stieltjes moment approach

Xiaojing $\mathrm{Xi}^{1}$, Marianito R. Rodrigo ${ }^{2}$ and Rogemar S. Mamon ${ }^{3}$

${ }^{1}$ Department of Applied Mathematics, University of Western Ontario, London, Ontario, Canada

${ }^{2}$ School of Mathematics and Applied Statistics, University of Wollongong, Wollongong, New South Wales, Australia

${ }^{3}$ Department of Statistical and Actuarial Sciences, University of Western Ontario, London, Ontario, Canada

We address the problem of recovering the time-dependent parameters of the Black-Scholes option pricing model when the underlying stock price dynamics are modelled by a finite-state, continuous-time Markov chain. The coupled system of Dupire-type partial differential equations is derived and formulated as an inverse Stieltjes moment problem. We provide numerical illustration on how to apply our method to simulated financial data. The accuracy of the model parameter estimation is examined and sensitivity analyses are included to study the behaviour of the estimated results when model parameters are varied.

\section{Contents}

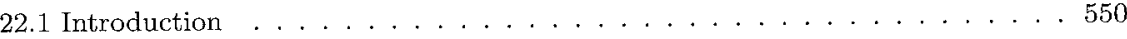
22.2 Regime-switching model setup . . . . . . . . . . . . . 552 22.2 Regine 554 22.4 Inverse Stieltjes moment problem . . . . . . . . . . . . . . 556 22.5 Numerical implementation and results . . . . . . . . . . . . 558 22.6 Conclusion $\ldots \ldots \ldots \ldots \ldots \ldots \ldots \ldots \ldots$

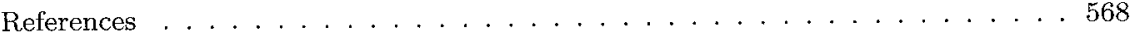




\subsection{Introduction}

A fundamental problem in financial mathematics is the recovery of model parameters given observed market prices. Volatility, for instance, is an important but unobservable parameter, whose estimate is necessary when pricing derivatives and enables us to understand price dynamics. Traders calculate implied volatilities from market data for option valuation, as well as using them as a guide to monitor the market's sentiments. In the present work, we focus on recovering the parameters of a regime-switching model from European call option prices.

A number of approaches have been proposed to deal with this type of problem. In a pioneering paper, Dupire [1] verifies empirically that different strikes and maturities lead to different implied volatilities for options on a given asset. Boyle and Thangaraj [2], as well as Andersen and BrothertonRatcliffe [3], obtain local implied volatilities by numerically implementing Dupire's equation. Rodrigo and Mamon [4] give a new expression for the volatility by deriving a semi-explicit solution of Dupire's equation. They also provide a different formula in [5], which makes use of the so-called inverse Stieltjes moment approach. Bouchouev and Isakov [6] reduce the identification of the volatility to an inverse parabolic problem with the final observation. Deng et al. [7] employ an optimal control framework with a new terminal condition to solve this kind of inverse problem.

Recently, considerable attention has been given to the use of regimeswitching models, or hidden Markov models (HMMs), in finance. In an HMM, the model parameters switch amongst unobservable states of the economy and are governed by a Markov process. A regime-switching volatility is a simple way to incorporate stochastic volatilities. It has the ability to capture long-term and fundamental changes in the economic mechanism that generates the data. Significant empirical evidence from the literature lends support for the appropriateness of regime-switching models. For instance, Chu et al. [8] advocate the use of these models to describe returns and volatility dynamics in the stock market. Turner et al. [9] argue that either the mean or variance, or both, may exhibit differences between two regimes. The investigation of Engel and Hamilton [10], Bekaert and Hodrick [11], and Engel and Hakkio [12] document regime switching in major foreign exchange rates. Dahlquist and Gray [13] and Ang and Bekaert [14] show that various foreign, short-term interest rates are well described by regime-switching models. Some applications of regime-switching models modulated by a hidden Markov chain can be found in the work of Elliott and Mamon [15], as well as in Elliott and Kopp [16].

Regime-switching models have achieved growing importance in various financial problems as they can capture a richer set of empirical and theoretical characteristics of a market. They have also enriched the developments in option pricing theory. For example, Elliott et al. [17] develop a method to price options based on a regime-switching random Esscher transform. In turn, this method was used by Ching et al. [18] to price exotic options under a hidden Markov model with long-range dependence in the states of an economy, which is known as a higher-order HMM. Mamon and Rodrigo [19] present closed-form solutions for European option values when the dynamics of both the short rate and the volatility of the underlying price process are modulated by a continuous-time Markov chain. Boyle and Draviam [20] derive the system of partial differential equations (PDEs) of Black-Scholes type that governs the dynamics of European options in a regime-switching framework and price exotic options by solving the coupled PDEs numerically. Duan et al. [21] develop a family of option pricing models which are based on the GARCH process and the variance-updating schemes also depend on a second factor orthogonal to asset innovations. Other works that feature regime-switching models in other applications include Siu et al. [22] for credit default swaps, Elliott and van der Hoek [23] for asset allocations, and Elliott and Mamon [15] for short-term interest rates.

The above studies in option pricing under a regime-switching framework serve as motivation for investigating the inverse problem of recovering the volatilities when they are governed by HMMs. There is a relatively limited amount of literature on estimating regime-switching parameters using market data. In this paper, we extend the inverse Stieltjes moment approach in [5] by assuming that the volatility of the underlying asset is governed by a continuous-time Markov chain. In this model, the unobservable parameters are the volatilities in each state and the intensity probabilities of the hidden Markov chain. We start with the well-known system of Black-Scholes-type PDEs and derive the coupled system of Dupire-type PDEs that governs the dynamics of European option prices.

The rest of the paper is organised as follows. In Section 22.2 , we recall the regime-switching model setup. In Section 22.3, we derive the system of Dupire-type PDEs describing the dynamics of European option prices under this setup. We formulate the inverse Stieltjes moment problem in Section 22.4, and also discuss how our proposed method could determine the model parameters. In Section 22.5, we exhibit an implementation to a set of "theoretical data" which were generated by solving the coupled 
Dupire-type PDEs. We conclude with a brief summary in Section 22.6.

\subsection{Regime-switching model setup}

We wish to value a European option within the standard Black-Scholes market with two basic securities consisting of a riskless asset (a bond whose value is $B_{t}$ at time $t \geq 0$ ) and a risky asset (a stock whose price is $S_{t}$ at time $t$ ). Moreover, we assume that the economic state of the world is modelled by a finite-state Markov chain $\mathbf{x}_{t}$ that evolves in continuous time. This implies that the bank rate process $r_{t}$ and the stock's volatility $\sigma_{t}$ and rate of return $\mu_{t}$ are governed by Markov chain dynamics.

Without loss of generality, we may take the state space of $\mathbf{x}_{t}$ to be the finite set $\left\{\mathbf{e}_{1}, \ldots, \mathbf{e}_{N}\right\}$ of canonical vectors in $\mathbb{R}^{N}$. Assume that $\mathbf{x}_{t}$ is homogeneous in time and has intensity matrix $\mathbf{A}=\left(a_{i j}\right)$, i.e.,

$$
a_{j i} \geq 0 \quad \text { for } j \neq i, \quad \sum_{i=1}^{N} a_{i j}=0 \quad \text { for each } j=1, \ldots, N .
$$

If $\mathbf{p}_{t}=E\left[\mathbf{x}_{t}\right]=\left(p_{t}^{1}, \ldots, p_{t}^{N}\right)^{*}$ where ${ }^{*}$ is the transpose operator, then $\mathbf{p}_{t}$ satisfies

$$
\frac{\mathrm{d} \mathbf{p}_{t}}{\mathrm{~d} t}=\mathbf{A} \mathbf{p}_{t}
$$

It can be shown [24] that $\mathbf{x}_{t}$ has a semi-martingale representation

$$
\mathbf{x}_{t}=\mathbf{x}_{0}+\int_{0}^{t} \mathbf{A} \mathbf{x}_{u} \mathrm{~d} u+\mathbf{M}_{t}
$$

where $\mathbf{M}_{t}$ is a martingale.

Suppose that $r_{t}=\left\langle\mathbf{r}, \mathbf{x}_{t}\right\rangle$ for some given vector $\mathbf{r}=\left(r_{1}, \ldots, r_{N}\right)^{*}$ in $\mathbb{R}^{N}$ with $r_{1}, \ldots, r_{N}>0$. Here, $\langle\cdot, \cdot\rangle$ denotes the usual inner product in $\mathbb{R}^{N}$. Then $\$ 1$ invested at time zero becomes

$$
B_{t}=e^{\int_{0}^{t} r_{u} \mathrm{~d} u}
$$

at time $t$. In addition, suppose that the rate of return $\mu_{t}$ and the volatility $\sigma_{t}$ depend on the state $\mathbf{x}_{t}$, i.e., there exist vectors $\boldsymbol{\mu}=\left(\mu_{1}, \ldots, \mu_{N}\right)^{*}$ and $\boldsymbol{\sigma}=\left(\sigma_{1}, \ldots, \sigma_{N}\right)^{*}$ in $\mathbb{R}^{N}$ (with $\mu_{i}, \sigma_{i}>0$ for all $i=1, \ldots, N$ ) such that $\mu_{t}=\left\langle\boldsymbol{\mu}, \mathbf{x}_{t}\right\rangle$ and $\sigma_{t}=\left\langle\boldsymbol{\sigma}, \mathbf{x}_{t}\right\rangle$. Then the dynamics of the stock is described by the stochastic differential equation

$$
\mathrm{d} S_{t}=\mu_{t} S_{t} \mathrm{~d} t+\sigma_{t} S_{t} \mathrm{~d} W_{t}
$$

where $W_{t}$ is a Brownian motion on a filtered probability space denoted by $\left(\Omega, \mathcal{F}, P,\left(\mathcal{F}_{t}\right)_{t \geq 0}\right)$ and $\left(\mathcal{F}_{t}\right)_{t \geq 0}$ is taken to be the natural filtration. It can be shown that $S_{t}$ is expressible as

$$
S_{t}=S_{0} e^{\int_{0}^{t}\left(\mu_{u}-\sigma_{u}^{2} / 2\right) \mathrm{d} u+\int_{0}^{t} \sigma_{u} \mathrm{~d} W_{u}}
$$

If the bond and stock dynamics are given by Eq. (22.1) and Eq. (22.2), respectively, and if at time $t \in[0, T]$ we have $S_{t}=S$ and $\mathbf{x}_{t}=\mathbf{x}$, then the price of a European call option with expiry $T$ and strike price $K$ is

$$
c(t, S, T, K, \mathbf{x})=E^{Q}\left[e^{-\int_{t}^{T} r_{u} \mathrm{~d} u}\left(S_{T}-K\right)^{+} \mid S_{t}=S, \mathbf{x}_{t}=\mathbf{x}\right]
$$

where $(z)^{+}=\max (z, 0)$ and $E^{Q}$ denotes the expectation evaluated under a risk-neutral measure $Q$. We remark that regime switching leads to an incomplete market, which can be completed by the introduction of ArrowDebreu securities [25] related to the cost of switching. Thus, in Eq. (22.3) we are assuming that we are already working under a risk-neutral measure $Q$. Just like in the classical Black-Scholes case, we assume that $\mu=r$ in the stock price dynamics under $Q$; hence the rate of return will not appear in Eq. (22.3). We do not rule out the dependence of the market price of risk on the state $x_{t}$ at time $t$. But, irrespective of whether or not we assume a special or functional form for the market price of risk that depends on $\mathbf{x}_{t}$, or some other more general dependence which we do not know, the information from the market should be implicitly reflected in the parameters that we want to estimate. That is, we do not know what the exact dependence is but what are important to us are the parameter estimates that should encapsulate this information.

Define $c_{i}(t, S, T, K)=c\left(t, S, T, K, \mathbf{e}_{i}\right)$ for each $i=1, \ldots, N$. We note that $r_{i}=\left\langle\mathbf{r}, \mathbf{e}_{i}\right\rangle$ and $\sigma_{i}=\left\langle\boldsymbol{\sigma}, \mathbf{e}_{i}\right\rangle$. It can be shown [19] that $c_{1}, \ldots, c_{N}$ satisfy a system of coupled PDEs of Black-Scholes type in the variables $t$ and $S$, namely

$$
\frac{\partial c_{i}}{\partial t}+\frac{1}{2} \sigma_{i}^{2} S^{2} \frac{\partial^{2} c_{i}}{\partial S^{2}}+r_{i} S \frac{\partial c_{i}}{\partial S}-r_{i} c_{i}+\sum_{j=1}^{N} a_{j i} c_{j}=0 \quad(i=1, \ldots, N)
$$

together with the terminal conditions

$$
c_{i}(T, S, T, K)=(S-K)^{+} \quad(i=1, \ldots, N)
$$

Let $\mathbf{c}=\left(c_{1}, \ldots, c_{N}\right)^{*}, \mathbf{\Sigma}=\operatorname{diag}\left(\sigma_{1}, \ldots, \sigma_{N}\right)$, and $\mathbf{R}=\operatorname{diag}\left(r_{1}, \ldots, r_{N}\right)$. Then Eq. (22.4), Eq. (22.5) can be recast in matrix form as

$$
\frac{\partial \mathbf{c}}{\partial t}+\frac{1}{2} S^{2} \mathbf{\Sigma}^{2} \frac{\partial^{2} \mathbf{c}}{\partial S^{2}}+S \mathbf{R} \frac{\partial \mathbf{c}}{\partial S}-\mathbf{R} \mathbf{c}+\mathbf{A}^{*} \mathbf{c}=\mathbf{0},
$$




$$
\mathbf{c}(T, S, T, K)=(S-K)^{+} \mathbf{1}
$$

respectively, where $\mathbf{0}$ is the $N$-dimensional zero vector and $\mathbf{1}$ is the $N$ dimensional vector all of whose components are equal to one.

Our aim here is to solve the inverse problem of recovering the parameters of the underlying model from market data. The inverse problem was first considered by Dupire [1], who showed that if the prices of a European call option were known for all strike prices and maturity dates, then the volatility surface can be recovered from market data. In our case, instead of a local volatility function, we wish to recover the volatility matrix $\boldsymbol{\Sigma}$, the transition intensity matrix $\mathbf{A}$, and the rate matrix $\mathbf{R}$.

It is important to note that actual market option prices are quoted for varying strikes and times to maturity. Thus, since we want to utilise a PDE-based approach to solve the inverse problem, we must first derive a system of PDEs similar to Eq. (22.6) but with the independent variables being the time to maturity and the strike price. In other words, we wish to obtain the analogue of Dupire's equation for the system of PDEs given in Eq. (22.6), which is the goal of the next section.

\subsection{Derivation of a system of Dupire-type PDEs}

First, we show that $c_{1}, \ldots, c_{N}$ are homogeneous functions of degree one with respect to $S$ and $K$, i.e.,

$$
c_{i}(t, \lambda S, T, \lambda K)=\lambda c_{i}(t, S, T, K) \quad(i=1, \ldots, N)
$$

for all $\lambda>0$. To prove Eq. (22.8), we will use a uniqueness argument by showing that $c_{i}(t, \lambda S, T, \lambda K)$ and $\lambda c_{i}(t, S, T, K)$ for all $i=1, \ldots, N$ satisfy the following final-value problem for $v(t, x, u, y)$ :

$$
\begin{gathered}
\frac{\partial v_{i}}{\partial t}+\frac{1}{2} \sigma_{i}^{2} x^{2} \frac{\partial^{2} v_{i}}{\partial x^{2}}+r_{i} x \frac{\partial v_{i}}{\partial x}-r_{i} v_{i}+\sum_{j=1}^{N} a_{j i} v_{j}=0 \quad(i=1, \ldots, N), \\
v_{i}(T, x, u, y)=\lambda(x-y)^{+} \quad(i=1, \ldots, N) .
\end{gathered}
$$

Let $c_{1}, \ldots, c_{N}$ be a solution of Eq. (22.4), Eq. (22.5). Take $v_{i}(t, x, u, y)=$ $\lambda c_{i}(t, S, T, K)$ where $x=S, u=T$, and $y=K$. Then it is easy to see that $v_{1}, \ldots, v_{N}$ satisfy Eq. (22.9), Eq. (22.10). Now take $v_{i}(t, x, u, y)=$ $c_{i}(t, S, T, K)$ where $x=S / \lambda, u=T$, and $y=K / \lambda$. Again, it is straightforward to verify that $v_{1}, \ldots, v_{N}$ satisfy Eq. (22.9), Eq. (22.10). Thus, the homogeneity condition Eq. (22.8) follows from the uniqueness of the solution of the final-value problem.

Invoking Euler's theorem on homogeneous functions, we obtain

$$
S \frac{\partial c_{i}}{\partial S}+K \frac{\partial c_{i}}{\partial K}=c_{i} \quad(i=1, \ldots, N) .
$$

Differentiating the above equation with respect to $S$ and $K$ gives

$$
S \frac{\partial^{2} c_{i}}{\partial S^{2}}=-K \frac{\partial^{2} c_{i}}{\partial S \partial K}, \quad K \frac{\partial^{2} c_{i}}{\partial K^{2}}=-S \frac{\partial^{2} c_{i}}{\partial K \partial S} \quad(i=1, \ldots, N),
$$

respectively. It follows that

$$
S^{2} \frac{\partial^{2} c_{i}}{\partial S^{2}}=K^{2} \frac{\partial^{2} c_{i}}{\partial K^{2}} \quad(i=1, \ldots, N)
$$

and Eq. (22.4) becomes

$$
\frac{\partial c_{i}}{\partial t}+\frac{1}{2} \sigma_{i}^{2} K^{2} \frac{\partial^{2} c_{i}}{\partial K^{2}}-r_{i} K \frac{\partial c_{i}}{\partial K}+\sum_{j=1}^{N} a_{j i} c_{j}=0 \quad(i=1, \ldots, N)
$$

with the same terminal condition Eq. (22.5). In matrix form we therefore have

$$
\begin{gathered}
\frac{\partial \mathbf{c}}{\partial t}+\frac{1}{2} K^{2} \mathbf{\Sigma}^{2} \frac{\partial^{2} \mathbf{c}}{\partial K^{2}}-K \mathbf{R} \frac{\partial \mathbf{c}}{\partial K}+\mathbf{A}^{*} \mathbf{c}=\mathbf{0} \\
\mathbf{c}(T, S, T, K)=(S-K)^{+} \mathbf{1}
\end{gathered}
$$

Finally, letting $\mathbf{c}(t, S, T, K)=\overline{\mathbf{c}}(u, S, K)$ where $u=T-t$ in Eq. (22.12), Eq. (22.13) yields

$$
\begin{gathered}
\frac{\partial \overline{\mathbf{c}}}{\partial u}=\frac{1}{2} K^{2} \boldsymbol{\Sigma}^{2} \frac{\partial^{2} \overline{\mathbf{c}}}{\partial K^{2}}-K \mathbf{R} \frac{\partial \overline{\mathbf{c}}}{\partial K}+\mathbf{A}^{*} \overline{\mathbf{c}} \\
\overline{\mathbf{c}}(0, S, K)=(S-K)^{+} \mathbf{1}
\end{gathered}
$$

respectively. Note that Eq. (22.14), Eq. (22.15) is now an initial-value problem. When $N=1$, we have

$$
\frac{\partial \bar{c}_{1}}{\partial u}=\frac{1}{2} \sigma_{1}^{2} K^{2} \frac{\partial^{2} \bar{c}_{1}}{\partial K^{2}}-r_{1} K \frac{\partial \bar{c}_{1}}{\partial K}
$$


. which is Dupire's equation with a constant the regime-switching case.

is the analogue of Dupire's equation for

\subsection{Inverse Stieltjes moment problem}

Having derived the system of PDEs in the appropriate independent variPolve the inverse problem of parameter ables $u$ and $K$, we now proced ties moment method first proposed in [5]. estimation via the inverse Stieltjes moment meth $\mathbf{c}(u, K)$ instead of

To simplify the ensuing notation, we shall write $\mathbf{c}(u, K)$ for some given $\overline{\mathbf{c}}(u, S, K)$. We will also assume for simplicity that $\mathbf{R}$ be easily extended to $r>0$, where $\mathbf{I}$ is the identity matrix; the method can be easily extend the call the more general case. As in [5], let us define the $n$th moment of the call price by

$$
m_{n}^{(i)}(u)=\int_{0}^{\infty} K^{n} c_{i}(u, K) \mathrm{d} K(i=1, \ldots, N),
$$

where $n$ is a nonnegative integer. Multiplying both sides of Eq. (22.14) by n

$$
\begin{aligned}
\int_{0}^{\infty} K^{n} \frac{\partial \mathbf{c}}{\partial u} \mathrm{~d} K= & \frac{1}{2} \int_{0}^{\infty} K^{n+2} \boldsymbol{\Sigma}^{2} \frac{\partial^{2} \mathbf{c}}{\partial K^{2}} \mathrm{~d} K-\int_{0}^{\infty} r K^{n+1} \frac{\partial \mathbf{c}}{\partial K} \mathrm{~d} K \\
& +\int_{0}^{\infty} K^{n} \mathbf{A}^{*} \mathbf{c} \mathrm{d} K
\end{aligned}
$$

tecays to zero sufficiently fast as $K \rightarrow \infty$, we deduce that

$$
\begin{aligned}
\int_{0}^{\infty} K^{n} \frac{\partial \mathbf{c}}{\partial u} \mathrm{~d} K & =\frac{\mathrm{d} \mathbf{m}_{n}}{\mathrm{~d} u} \\
\int_{0}^{\infty} K^{n+1} \frac{\partial \mathbf{c}}{\partial K} \mathrm{~d} K & =-(n+1) \mathbf{m}_{n} \\
\int_{0}^{\infty} K^{n+2} \frac{\partial^{2} \mathbf{c}}{\partial K^{2}} \mathrm{~d} K & =(n+1)(n+2) \mathbf{m}_{n}
\end{aligned}
$$

Thus, Eq. (22.17) simplifies to

$$
\frac{\mathrm{d} \mathbf{m}_{n}}{\mathrm{~d} u}-r(n+1) \mathbf{m}_{n}=\left[\frac{1}{2}(n+1)(n+2) \boldsymbol{\Sigma}^{2}+\mathbf{A}^{*}\right] \mathbf{m}_{n} .
$$

Considering any $N$ consecutive moments gives

$$
\begin{aligned}
\frac{\mathrm{d} \mathbf{m}_{n}}{\mathrm{~d} u}-r(n+1) \mathbf{m}_{n}= & {\left[\frac{(n+1)(n+2)}{2} \boldsymbol{\Sigma}^{2}+\mathbf{A}^{*}\right] \mathbf{m}_{n}, } \\
\frac{\mathrm{d} \mathbf{m}_{n+1}}{\mathrm{~d} u}-r(n+2) \mathbf{m}_{n+1}= & {\left[\frac{(n+2)(n+3)}{2} \boldsymbol{\Sigma}^{2}+\mathbf{A}^{*}\right] \mathbf{m}_{n+1}, } \\
& \vdots \\
\frac{\mathrm{d} \mathbf{m}_{n+N-1}}{\mathrm{~d} u}-r(n+N) \mathbf{m}_{n+N-1}= & {\left[\frac{(n+N)(n+N+1)}{2} \Sigma^{2}+\mathbf{A}^{*}\right] \mathbf{m}_{n+N-1} . }
\end{aligned}
$$

Since the option prices are assumed to be observed, the moments are also $\mathbf{A}$ and $\boldsymbol{\Sigma}$. Given that $\mathbf{A}$ is an intensity known and we wish to estimate $\mathbf{A}$ and $\boldsymbol{\Sigma}$. Given that $\mathbf{A}$ is an inten matrix, each of its $N$ diagonal entries, say $a_{i i}$, is expressible as a sum of the entries in the $i$ th column, so there are essentially $N^{2}-N$ unknown entries of A Together with the $N$ unknown diagonal entries of $\Sigma$, we therefore of A. Together with the $N$ un to estimate. Note that Eq. (22.18) is a linear have a total of $N^{2}$ parans in $N^{2}$ unknowns.

system of $N^{2}$ equation basic idea, let us take $N=2$. Then Eq. (22.18) gives

$$
\begin{aligned}
& \left(\begin{array}{cccc}
\frac{(n+1)(n+2)}{2} m_{n}^{(1)} & 0 & m_{n}^{(2)}-m_{n}^{(1)} & 0 \\
0 & \frac{(n+1)(n+2)}{2} m_{n}^{(2)} & 0 & m_{n}^{(1)}-m_{n}^{(2)} \\
\frac{(n+2)(n+3)}{2} m_{n+1}^{(1)} & 0 & m_{n+1}^{(2)}-m_{n+1}^{(1)} & 0 \\
0 & \frac{(n+2)(n+3)}{2} m_{n+1}^{(2)} & 0 & m_{n+1}^{(1)}-m_{n+1}^{(2)}
\end{array}\right) \\
& \quad \times\left(\begin{array}{c}
\sigma_{1}^{2} \\
\sigma_{2}^{2} \\
a_{21} \\
a_{12}
\end{array}\right)=\left(\begin{array}{l}
\frac{\mathrm{d} m_{n}^{(1)}}{\mathrm{d} u}-r(n+1) m_{n}^{(1)} \\
\frac{\mathrm{d} m_{n}^{(2)}}{\mathrm{d} u}-r(n+1) m_{n}^{(2)} \\
\frac{\mathrm{d} m_{n+1}^{(1)}}{\mathrm{d} u}-r(n+2) m_{n+1}^{(1)} \\
\frac{\mathrm{d} m_{n+1}^{(2)}}{\mathrm{d} u}-r(n+2) m_{n+1}^{(2)}
\end{array}\right) .
\end{aligned}
$$

Note that from Eq. (22.15) we see that

$$
\mathbf{m}_{n}(0)=\int_{0}^{\infty} K^{n}(S-K)^{+} \mathbf{1} \mathrm{d} K=\frac{S^{n+2}}{(n+1)(n+2)} \mathbf{1} .
$$

Let

$$
M_{n}^{(i)}(u)=\int_{0}^{u} m_{n}^{(i)}(s) \mathrm{d} s \quad(i=1,2)
$$


To incorporate the initial conditions, we integrate Eq. (22.19) over $[0, u]$ with respect to a dummy variable $s$ to get

$$
\begin{aligned}
& \left(\begin{array}{cccc}
\frac{(n+1)(n+2)}{2} M_{n}^{(1)} & 0 & M_{n}^{(2)}-M_{n}^{(1)} & 0 \\
0 & \frac{(n+1)(n+2)}{2} M_{n}^{(2)} & 0 & M_{n}^{(1)}-M_{n}^{(2)} \\
\frac{(n+2)(n+3)}{2} M_{n+1}^{(1)} & 0 & M_{n+1}^{(2)}-M_{n+1}^{(1)} & 0 \\
0 & \frac{(n+2)(n+3)}{2} M_{n+1}^{(2)} & 0 & M_{n+1}^{(1)}-M_{n+1}^{(2)}
\end{array}\right) \\
& \times\left(\begin{array}{c}
\sigma_{1}^{2} \\
\sigma_{2}^{2} \\
a_{21} \\
a_{12}
\end{array}\right)=\left(\begin{array}{l}
m_{n}^{(1)}-\frac{S^{n+2}}{(n+1)(n+2)}-r(n+1) M_{n}^{(1)} \\
m_{n}^{(2)}-\frac{S^{n+2}}{(n+1)(n+2)}-r(n+1) M_{n}^{(2)} \\
m_{n+1}^{(1)}-\frac{S^{n+3}}{(n+2)(n+3)}-r(n+2) M_{n+1}^{(1)} \\
m_{n+1}^{(2)}-\frac{S^{n+3}}{(n+2)(n+3)}-r(n+2) M_{n+1}^{(2)}
\end{array}\right) .
\end{aligned}
$$

In summary, given $c_{1}(u, K)$ and $c_{2}(u, K)$ where $0 \leq u \leq T$ and $K \geq 0$, we compute $m_{n}^{(1)}, m_{n}^{(2)}, m_{n+1}^{(1)}$, and $m_{n+1}^{(2)}$ (for a fixed nonnegative integer $n$ ), as well as their integrals over $[0, u]$ for some $u$ in $[0, T]$. We then solve the linear system Eq. (22.20) for the unknown parameters $\sigma_{1}, \sigma_{2}, a_{21}$, and $a_{12}$. Note that $a_{11}=-a_{21}$ and $a_{22}=-a_{12}$ by the definition of $\mathbf{A}$. In addition, the choice of $u$ in $[0, T]$ should not matter since in this framework $\mathbf{A}$ and $\boldsymbol{\Sigma}$ are constant matrices.

\subsection{Numerical implementation and results}

To test the accuracy of the inverse Stieltes moment method, we need to have "observed" option prices. The "observed" option prices can be taken to be the solution generated by the initial-value problem Eq. (22.14), Eq. (22.15) (after specifying some matrices $\mathbf{A}$ and $\boldsymbol{\Sigma}$ ). Then we try to recover $\mathbf{A}$ and $\boldsymbol{\Sigma}$ using the moment method.

As a trial run, suppose that $\mathbf{A}=\mathbf{0}$, i.e., there is no switching among regimes. Then Eq. (22.14) reduces to a system of uncoupled Dupire equations. Hence, each component of $\mathbf{c}$ solves Dupire's equation, i.e., if $\mathbf{c}=\left(c_{1}, \ldots, c_{N}\right)^{*}$, then

$$
\begin{aligned}
& c_{i}(u, K)=S \Phi\left(d_{1}^{(i)}(u, K)\right)-K e^{-r u} \Phi\left(d_{2}^{(i)}(u, K)\right) \quad(i=1, \ldots, N), \\
& d_{1}^{(i)}(u, K)=\frac{\log (S / K)+\left(r+\sigma_{i}^{2} / 2\right) u}{\sigma_{i} \sqrt{u}} \\
& d_{2}^{(i)}(u, K)=\frac{\log (S / K)+\left(r-\sigma_{i}^{2} / 2\right) u}{\sigma_{i} \sqrt{u}}
\end{aligned}
$$

where $\Phi$ denotes the cumulative distribution function of a standard normal variable. Now we assume that $r=0.02, S=20, u=0, T=1, \sigma_{1}=$ 0.1 and $\sigma_{2}=0.3$. We take 50 values for strike price ranging from 0 to 60 and 100 values for the time to maturity ranging from 0 to 1 . Then the moments and their integrals are calculated and momment method in Eq. $(22.20)$ is applied. The estimated parameters are: $a_{21}=6.67 \times 10^{(-4)}$, $a_{12}=3.15 \times 10^{(-4)}, \sigma_{1}=0.1$ and $\sigma_{2}=0.2957$.

However, for the more realistic case $\mathbf{A} \neq \mathbf{0}$, there is no known explicit solution of Eq. (22.14), Eq. (22.15) in general. So we will have to solve this problem numerically to generate the "observed" option prices. This implies that we have to truncate the interval $(0, \infty)$ to some finite interval $\left(0, K_{\max }\right)$ where $K_{\max }>0$, and then impose reasonable boundary conditions for c at $K=0$ and $K=K_{\max }$. If the right endpoint $K_{\max }$ is sufficiently large, and recalling that each component of $\mathrm{c}$ tends to zero as $K \rightarrow \infty$, then we can assign a positive but small value to each component. However, the boundary condition at the left endpoint $K=0$ is not clear. When $N=1$, the Black-Scholes formula evaluated at $K=0$ gives $S$ for the call price. For $N>1$, it is not certain whether each component of $\mathbf{c}$ will also have the value $S$. To get around this problem, we will solve Eq. (22.14), Eq. (22.15) numerically for $K \in\left[0, K_{\max }\right]$ and $u \in[0, T]$ by formulating an explicit method with implicit boundary conditions.

Discretise the variables by

$$
u \simeq u^{i}, \quad K \simeq K^{j}, \quad \mathbf{c} \simeq \mathbf{c}^{i, j}=\left(c_{1}^{i, j}, c_{2}^{i, j}\right)^{*}
$$

where

$$
u^{i}=i \Delta u, \quad \Delta u=\frac{T}{I} \quad(i=0, \ldots, I)
$$

and

$$
K^{j}=j \Delta K, \quad \Delta K=\frac{K_{\max }}{J} \quad(j=0, \ldots, J) .
$$

Using an explicit scheme, we discretise Eq. (22.14) to get

$$
\begin{aligned}
\frac{c_{1}^{i+1, j}-c_{1}^{i, j}}{\Delta u}= & \frac{1}{2} \sigma_{1}^{2}\left(K^{j}\right)^{2} \frac{c_{1}^{i, j-1}-2 c_{1}^{i, j}+c_{1}^{i, j+1}}{(\Delta K)^{2}}-r K^{j} \frac{c_{1}^{i, j+1}-c_{1}^{i, j}}{\Delta K} \\
& +a_{11} c_{1}^{i, j}+a_{21} c_{2}^{i, j}, \\
\frac{c_{2}^{i+1, j}-c_{2}^{i, j}}{\Delta u}= & \frac{1}{2} \sigma_{2}^{2}\left(K^{j}\right)^{2} \frac{c_{2}^{i, j-1}-2 c_{2}^{i, j}+c_{2}^{i, j+1}}{(\Delta K)^{2}}-r K^{j} \frac{c_{2}^{i, j+1}-c_{2}^{i, j}}{\Delta K} \\
& +a_{12} c_{1}^{i, j}+a_{22} c_{2}^{i, j}
\end{aligned}
$$


This is equivalent to

$$
\begin{aligned}
\mathbf{c}^{i+1, j}= & \mathbf{c}^{i, j}+\frac{1}{2} \frac{\Delta u}{(\Delta K)^{2}}\left(K^{j}\right)^{2} \mathbf{\Sigma}^{2}\left(\mathbf{c}^{i, j-1}-2 \mathbf{c}^{i, j}+\mathbf{c}^{i, j+1}\right) \\
& -\frac{r \Delta u}{\Delta K} K^{j}\left(\mathbf{c}^{i, j+1}-\mathbf{c}^{i, j}\right)+\Delta u \mathbf{A}^{*} \mathbf{c}^{i, j}
\end{aligned}
$$

for all $i=1, \ldots, I-1$ and $j=1, \ldots, J-1$. This solves for the option prices at time $i+1$ in the open interval $\left(0, K_{\max }\right)$ using the option prices calculated at time $i$ over the closed interval $\left[0, K_{\max }\right]$. The initial condition is determined by

$$
\mathbf{c}^{0, j}=\left(S-K^{j}\right)^{+} \mathbf{1} \quad(j=0, \ldots, J)
$$

which includes the values at both endpoints.

To determine the boundary values at $K=0$, we use second-order Taylor expansions at $(u, 0)$ in the continuous variables, i.e.,

$$
\begin{gathered}
\mathbf{c}(u, \Delta K) \simeq \mathbf{c}(u, 0)+(\Delta K) \frac{\partial \mathbf{c}}{\partial K}(u, 0)+\frac{1}{2}(\Delta K)^{2} \frac{\partial^{2} \mathbf{c}}{\partial K^{2}}(u, 0), \\
\mathbf{c}(u, 2 \Delta K) \simeq \mathbf{c}(u, 0)+(2 \Delta K) \frac{\partial \mathbf{c}}{\partial K}(u, 0)+\frac{1}{2}(2 \Delta K)^{2} \frac{\partial^{2} \mathbf{c}}{\partial K^{2}}(u, 0), \\
\mathbf{c}(u, 3 \Delta K) \simeq \mathbf{c}(u, 0)+(3 \Delta K) \frac{\partial \mathbf{c}}{\partial K}(u, 0)+\frac{1}{2}(3 \Delta K)^{2} \frac{\partial^{2} \mathbf{c}}{\partial K^{2}}(u, 0) .
\end{gathered}
$$

Define

$$
\boldsymbol{\alpha}_{L}(u)=\frac{\partial \mathbf{c}}{\partial K}(u, 0), \quad \boldsymbol{\beta}_{L}(u)=\frac{\partial^{2} \mathbf{c}}{\partial K^{2}}(u, 0)
$$

Then in discretised variables we get

$$
\begin{aligned}
& \mathbf{c}^{i, 1}=\mathbf{c}^{i, 0}+\Delta K \boldsymbol{\alpha}_{L}\left(u^{i}\right)+\frac{1}{2}(\Delta K)^{2} \boldsymbol{\beta}_{L}\left(u^{i}\right), \\
& \mathbf{c}^{i, 2}=\mathbf{c}^{i, 0}+2 \Delta K \boldsymbol{\alpha}_{L}\left(u^{i}\right)+2(\Delta K)^{2} \boldsymbol{\beta}_{L}\left(u^{i}\right), \\
& \mathbf{c}^{i, 3}=\mathbf{c}^{i, 0}+3 \Delta K \boldsymbol{\alpha}_{L}\left(u^{i}\right)+\frac{9}{2}(\Delta K)^{2} \boldsymbol{\beta}_{L}\left(u^{i}\right),
\end{aligned}
$$

valid for all $i=1, \ldots, I$. In matrix form, this is the same as

$$
\left(\begin{array}{ccc}
1 & \Delta K & \frac{1}{2}(\Delta K)^{2} \\
1 & 2 \Delta K & 2(\Delta K)^{2} \\
1 & 3 \Delta K & \frac{9}{2}(\Delta K)^{2}
\end{array}\right)\left(\begin{array}{c}
c_{1}^{i, 0} \\
\alpha_{L}^{(1)}\left(u^{i}\right) \\
\beta_{L}^{(1)}\left(u^{i}\right)
\end{array}\right)=\left(\begin{array}{c}
c_{1}^{i, 1} \\
c_{1}^{i, 2} \\
c_{1}^{i, 3}
\end{array}\right)
$$

and

$$
\left(\begin{array}{ccc}
1 & \Delta K & \frac{1}{2}(\Delta K)^{2} \\
1 & 2 \Delta K & 2(\Delta K)^{2} \\
1 & 3 \Delta K & \frac{9}{2}(\Delta K)^{2}
\end{array}\right)\left(\begin{array}{c}
c_{2}^{i, 0} \\
\alpha_{L}^{(2)}\left(u^{i}\right) \\
\beta_{L}^{(2)}\left(u^{i}\right)
\end{array}\right)=\left(\begin{array}{c}
c_{2}^{i, 1} \\
c_{2}^{i, 2} \\
c_{2}^{i, 3}
\end{array}\right) .
$$

Note that the vectors $\boldsymbol{\alpha}_{L}\left(u^{i}\right)$ and $\boldsymbol{\beta}_{L}\left(u^{i}\right)$ are not known, which is why we need to solve these two linear systems to obtain the really desired quantity $\mathbf{c}^{i, 0}$. Moreover, $\mathbf{c}^{i, 1}, \mathbf{c}^{i, 2}$, and $\mathbf{c}^{i, 3}$ on the right-hand sides are known if we first solve the PDEs in the interior $\left(0, K_{\max }\right)$.

Similarly, at the right boundary point $K=K_{\max }$, we expand

$$
\begin{aligned}
\mathbf{c}\left(u, K_{\max }-\Delta K\right) \simeq & \mathbf{c}\left(u, K_{\max }\right)+(-\Delta K) \frac{\partial \mathbf{c}}{\partial K}\left(u, K_{\max }\right) \\
& +\frac{1}{2}(-\Delta K)^{2} \frac{\partial^{2} \mathbf{c}}{\partial K^{2}}\left(u, K_{\max }\right), \\
\mathbf{c}\left(u, K_{\max }-2 \Delta K\right) \simeq & \mathbf{c}\left(u, K_{\max }\right)+(-2 \Delta K) \frac{\partial \mathbf{c}}{\partial K}\left(u, K_{\max }\right) \\
& +\frac{1}{2}(-2 \Delta K)^{2} \frac{\partial^{2} \mathbf{c}}{\partial K^{2}}\left(u, K_{\max }\right), \\
\mathbf{c}\left(u, K_{\max }-3 \Delta K\right) \simeq & \mathbf{c}\left(u, K_{\max }\right)+(-3 \Delta K) \frac{\partial \mathbf{c}}{\partial K}\left(u, K_{\max }\right) \\
& +\frac{1}{2}(-3 \Delta K)^{2} \frac{\partial^{2} \mathbf{c}}{\partial K^{2}}\left(u, K_{\max }\right) .
\end{aligned}
$$

Defining

$$
\boldsymbol{\alpha}_{R}(u)=\frac{\partial \mathbf{c}}{\partial K}\left(u, K_{\max }\right), \quad \boldsymbol{\beta}_{R}(u)=\frac{\partial^{2} \mathbf{c}}{\partial K^{2}}\left(u, K_{\max }\right),
$$

we obtain

$$
\begin{aligned}
& \mathbf{c}^{i, J-1}=\mathbf{c}^{i, J}-\Delta K \boldsymbol{\alpha}_{R}\left(u^{i}\right)+\frac{1}{2}(\Delta K)^{2} \boldsymbol{\beta}_{R}\left(u^{i}\right), \\
& \mathbf{c}^{i, J-2}=\mathbf{c}^{i, J}-2 \Delta K \boldsymbol{\alpha}_{R}\left(u^{i}\right)+2(\Delta K)^{2} \boldsymbol{\beta}_{R}\left(u^{i}\right), \\
& \mathbf{c}^{i, J-3}=\mathbf{c}^{i, J}-3 \Delta K \boldsymbol{\alpha}_{R}\left(u^{i}\right)+\frac{9}{2}(\Delta K)^{2} \boldsymbol{\beta}_{R}\left(u^{i}\right),
\end{aligned}
$$

or, in matrix form

$$
\left(\begin{array}{ccc}
1 & -\Delta K & \frac{1}{2}(\Delta K)^{2} \\
1 & -2 \Delta K & 2(\Delta K)^{2} \\
1 & -3 \Delta K & \frac{9}{2}(\Delta K)^{2}
\end{array}\right)\left(\begin{array}{c}
c_{1}^{i, J} \\
\alpha_{R}^{(1)}\left(u^{i}\right) \\
\beta_{R}^{(1)}\left(u^{i}\right)
\end{array}\right)=\left(\begin{array}{c}
c_{1}^{i, J-1} \\
c_{1}^{i, J-2} \\
c_{1}^{i, J-3}
\end{array}\right)
$$


and

$$
\left(\begin{array}{ccc}
1-\Delta K & \frac{1}{2}(\Delta K)^{2} \\
1-2 \Delta K & 2(\Delta K)^{2} \\
1-3 \Delta K & \frac{9}{2}(\Delta K)^{2}
\end{array}\right)\left(\begin{array}{c}
c_{2}^{i, J} \\
\alpha_{R}^{(2)}\left(u^{i}\right) \\
\beta_{R}^{(2)}\left(u^{i}\right)
\end{array}\right)=\left(\begin{array}{c}
c_{2}^{i, J-1} \\
c_{2}^{i, J-2} \\
c_{2}^{i, J-3}
\end{array}\right)
$$

As before, the vectors $\boldsymbol{\alpha}_{R}\left(u^{i}\right)$ and $\boldsymbol{\beta}_{R}\left(u^{i}\right)$ are not known, so we solve these two linear systems to obtain the really desired quantity $\mathbf{c}^{i, J}$. Moreover $\mathbf{c}^{i, J-1}, \mathbf{c}^{i, J-2}$, and $\mathbf{c}^{i, J-3}$ on the right-hand sides are known if we first solve the PDEs in the interior $\left(0, K_{\max }\right)$.

Summarising, the explicit algorithm incorporating implicit boundary conditions that we propose can be formulated as follows:

(1) Set

$$
\mathbf{c}^{0, j}=\left(S-K^{j}\right)^{+} \mathbf{1} \quad(j=0, \ldots, J) .
$$

(2) For all $i=0, \ldots, I-1$ do

(a) For all $j=1, \ldots, J-1$ do

$$
\begin{aligned}
\mathbf{c}^{i+1, j}= & \mathbf{c}^{i, j}+\frac{1}{2} \frac{\Delta u}{(\Delta K)^{2}}\left(K^{j}\right)^{2} \Sigma^{2}\left(\mathbf{c}^{i, j-1}-2 \mathbf{c}^{i, j}+\mathbf{c}^{i, j+1}\right) \\
& -\frac{r \Delta u}{\Delta K} K^{j}\left(\mathbf{c}^{i, j+1}-\mathbf{c}^{i, j}\right)+\Delta u \mathbf{A}^{*} \mathbf{c}^{i, j}
\end{aligned}
$$

(b) Solve

$$
\left(\begin{array}{ccc}
1 & \Delta K & \frac{1}{2}(\Delta K)^{2} \\
1 & 2 \Delta K & 2(\Delta K)^{2} \\
1 & 3 \Delta K & \frac{9}{2}(\Delta K)^{2}
\end{array}\right)\left(\begin{array}{c}
c_{1}^{i+1,0} \\
\alpha_{L}^{(1)}\left(u^{i+1}\right) \\
\beta_{L}^{(1)}\left(u^{i+1}\right)
\end{array}\right)=\left(\begin{array}{c}
c_{1}^{i+1,1} \\
c_{1}^{i+1,2} \\
c_{1}^{i+1,3}
\end{array}\right)
$$

and

$$
\left(\begin{array}{ccc}
1 & \Delta K & \frac{1}{2}(\Delta K)^{2} \\
1 & 2 \Delta K & 2(\Delta K)^{2} \\
1 & 3 \Delta K & \frac{9}{2}(\Delta K)^{2}
\end{array}\right)\left(\begin{array}{c}
c_{2}^{i+1,0} \\
\alpha_{L}^{(2)}\left(u^{i+1}\right) \\
\beta_{L}^{(2)}\left(u^{i+1}\right)
\end{array}\right)=\left(\begin{array}{c}
c_{2}^{i+1,1} \\
c_{2}^{i+1,2} \\
c_{2}^{i+1,3}
\end{array}\right)
$$

for $\mathbf{c}^{i+1,0}=\left(c_{1}^{i+1,0}, c_{2}^{i+1,0}\right)^{*}$.

(c) Solve

$$
\left(\begin{array}{ccc}
1 & -\Delta K & \frac{1}{2}(\Delta K)^{2} \\
1 & -2 \Delta K & 2(\Delta K)^{2} \\
1 & -3 \Delta K & \frac{9}{2}(\Delta K)^{2}
\end{array}\right)\left(\begin{array}{c}
c_{1}^{i+1, J} \\
\alpha_{R}^{(1)}\left(u^{i+1}\right) \\
\beta_{R}^{(1)}\left(u^{i+1}\right)
\end{array}\right)=\left(\begin{array}{c}
c_{1}^{i+1, J-1} \\
c_{1}^{i+1, J-2} \\
c_{1}^{i+1, J-3}
\end{array}\right)
$$

and

$$
\left(\begin{array}{ccc}
1 & -\Delta K & \frac{1}{2}(\Delta K)^{2} \\
1 & -2 \Delta K & 2(\Delta K)^{2} \\
1 & -3 \Delta K & \frac{9}{2}(\Delta K)^{2}
\end{array}\right)\left(\begin{array}{c}
c_{2}^{i+1, J} \\
\alpha_{R}^{(2)}\left(u^{i+1}\right) \\
\beta_{R}^{(2)}\left(u^{i+1}\right)
\end{array}\right)=\left(\begin{array}{c}
c_{2}^{i+1, J-1} \\
c_{2}^{i+1, J-2} \\
c_{2}^{i+1, J-3}
\end{array}\right)
$$$$
\text { for } \mathbf{c}^{i+1, J}=\left(c_{1}^{i+1, J}, c_{2}^{i+1, J}\right)^{*} \text {. }
$$

A stability criterion for this scheme is

$$
\Delta u \leq(\Delta K)^{2} \min \left(\frac{1}{\sigma_{1}^{2}}, \frac{1}{\sigma_{2}^{2}}\right) .
$$

Although explicit schemes are generally slower than implicit schemes, the Although explicit sorer since programming is straightforward for the former compared to the linear system to be solved for the implicit scheme is not anymore tridi-
the ling agonal.

In the following simulations, we take the parameter values to be $r=$ $0.02, S=20, u=0, T=1, K_{\max }=60, \sigma_{1}=0.1$, and $\sigma_{2}=0.3$. We use 1200 nodes to discretize time axes and 120 nodes to discritize strike axes, i.e., $\Delta u=\frac{1}{1200}$ and $\Delta K=0.5$, in solving the PDEs (22.14), (22.15) numerically. The numerical solution contains a large size of dataset which usually does not exist in practice; in reality there is only a set of small data points corresponding to time and strike nodes. In our example, we pick 13-time and 21-strike nodes from the solutions and these prices are pick 13-time inverse Stieltjes moment approach. Next, in order to calculate the truncated moments and their integrals accurately, we interpolate the call prices from time to maturity- and strike-direction. The size of the dataset increases to 500 by 500 points after interpolation. The a Matlab built-in function called Piecewise Cubic Hermite Here, we use a Matlab built-in function called Plation procedure. Note that in general that the market data is not equally spaced through time that in gerity and strike prices. Therefore, the number of nodes interpolated between two prices depends on the differences in time to maturity and strike price of the two prices. Finally we solve the algebraic system (22.20) for the "unknown" parameters.

First, let us suppose that the intensity matrix $\mathbf{A}$ is of the form

$$
\mathbf{A}=\left(\begin{array}{cc}
-\lambda & \lambda \\
\lambda & -\lambda
\end{array}\right)
$$

where $\lambda>0$. In Table 22.1, we present the estimated parameters for different values of $\lambda$ and $n$. Additionally, we evaluate the errors for the estimated 
Table 22.1. Example 1: Estimated parameters for different $\lambda$ and $n$ with $\sigma_{\mathbf{1}}=0.1$ and $\sigma_{2}=0.3$

\begin{tabular}{|c|c|c|c|c|}
\hline \multirow[b]{2}{*}{$\lambda$} & \multirow{2}{*}{$\begin{array}{l}\text { Estimated } \\
\text { parameter }\end{array}$} & \multicolumn{3}{|c|}{$n$} \\
\hline & & 2 & 3 & 4 \\
\hline 0.25 & $\begin{array}{c}\left(\lambda_{1}, \lambda_{2}\right) \\
\left(\sigma_{1}, \sigma_{2}\right) \\
\left(\mathrm{rmse}_{1}, \mathrm{rmse}_{2}\right)\end{array}$ & $\begin{array}{l}(0.2567,0.2240) \\
(0.0977,0.3002) \\
(0.0021,0.0058)\end{array}$ & $\begin{array}{l}(0.2523,0.1995) \\
(0.0987,0.2989) \\
(0.0013,0.0069)\end{array}$ & $\begin{array}{c}(0.2498,0.1477) \\
(0.0994,0.2964) \\
\left(7.70 \times 10^{-4}, 0.0094\right)\end{array}$ \\
\hline 0.5 & $\begin{array}{c}\left(\lambda_{1}, \lambda_{2}\right) \\
\left(\sigma_{1}, \sigma_{2}\right) \\
\left(\mathrm{rmse}_{1}, \mathrm{rmse}_{2}\right)\end{array}$ & $\begin{array}{l}(0.5073,0.4686) \\
(0.0978,0.3001) \\
(0.0013,0.0051)\end{array}$ & $\begin{array}{c}(0.5016,0.4420) \\
(0.0989,0.2989) \\
\left(6.49 \times 10^{-4}, 0.0060\right)\end{array}$ & $\begin{array}{c}(0.4982,0.3844) \\
(0.0997,0.2964) \\
\left(1.7 \times 10^{-4}, 0.0083\right)\end{array}$ \\
\hline 1 & $\begin{array}{c}\left(\lambda_{1}, \lambda_{2}\right) \\
\left(\sigma_{1}, \sigma_{2}\right) \\
\left(\mathrm{rmse}_{1}, \mathrm{rmse}_{2}\right)\end{array}$ & $\begin{array}{c}(1.0088,0.9570) \\
(0.0981,0.2999) \\
\left(8.4 \times 10^{-4}, 0.0042\right)\end{array}$ & $\begin{array}{c}(0.9995,0.9205) \\
(0.0994,0.2988) \\
\left(7.22 \times 10^{-4}, 0.0049\right)\end{array}$ & $\begin{array}{c}(0.9939,0.8571) \\
(0.1002,0.2964) \\
\left(8.87 \times 10^{-4}, 0.0065\right)\end{array}$ \\
\hline 2 & $\begin{array}{c}\left(\lambda_{1}, \lambda_{2}\right) \\
\left(\sigma_{1}, \sigma_{2}\right) \\
\left(\mathrm{rmse}_{1}, \mathrm{rmse}_{2}\right)\end{array}$ & $\begin{array}{l}(2.0094,1.9369) \\
(0.0987,0.2999) \\
(0.0015,0.0034)\end{array}$ & $\begin{array}{l}(1.9900,1.9001) \\
(0.1004,0.2990) \\
(0.0015,0.0019)\end{array}$ & $\begin{array}{l}(1.9782,0.19101 \\
(0.1015,0.2968) \\
(0.0020,0.0047)\end{array}$ \\
\hline 5 & $\begin{array}{c}\left(\lambda_{1}, \lambda_{2}\right) \\
\left(\sigma_{1}, \sigma_{2}\right) \\
\left(\mathrm{rmse}_{1}, \mathrm{rmse}_{2}\right)\end{array}$ & $\begin{array}{l}(4.9470,5.0349) \\
(0.1021,0.3017) \\
(0.0043,0.0051)\end{array}$ & $\begin{array}{l}(4.9522,4.8768) \\
(0.1047,0.3007) \\
(0.0041,0.0046)\end{array}$ & $\begin{array}{l}(4.8405,4.8238) \\
(0.1061,0.2992) \\
(0.0024,0.0028)\end{array}$ \\
\hline 8 & $\begin{array}{c}\left(\lambda_{1}, \lambda_{2}\right) \\
\left(\sigma_{1}, \sigma_{2}\right) \\
\left(\text { rmse }_{1}, \text { rmse }_{2}\right)\end{array}$ & $\begin{array}{l}(7.7259,8.6577) \\
(0.1069,0.3062) \\
(0.0162,0.0179)\end{array}$ & $\begin{array}{l}(7.5802,8.3862) \\
(0.1102,0.3041) \\
(0.0143,0.0160)\end{array}$ & $\begin{array}{l}(7.5168,8.1685) \\
(0.1116,0.3024) \\
(0.0120,0.0139)\end{array}$ \\
\hline
\end{tabular}

option prices using Root Mean Square Error (RMSE), which helps us to analyse the sensitivity of $\lambda$ and $n$. From Eq. (22.20), the estimated parameters should be independent of $n$. The error, which occurs by sovling the Dupire PDEs, certainly affects the calculation. To rectify this to some extent, we utilise larger degrees of the moment, i.e., $n \geq 2$, which would put more weight on option prices in the calculation of moments and consequently reduce the impact of the errors in the Dupire PDEs. In this example, the column corresponding to $n=2$ shows very good agreement between the estimated values and the actual values assigned when $\lambda \leq 2$. However, if the value of $n$ is too large, higher calculation error occurs when the moments are calculated, and it also affects the estimated results. As can be observed, in the case of $\lambda \leq 2$, RMSE is larger when higher $n$ is used. Furthermore, the accuracy of the estimation is also affected by the value of the intensity rate. As the intensity rate increases, the market tends to switch more frequently between the states and thus, it is more difficult to capture the information from the market data reducing the accuracy of the estimation. Again, this problem can be corrected by using larger degrees of the moment. Therefore, when $\lambda \geq 2$, RMSE is smaller when higher $n$ is used.

In our second numerical experiment, we assume different intensity rates for state 1 and state 2 , so the intensity matrix is of the form

$$
\mathbf{A}=\left(\begin{array}{cc}
-\lambda_{1} & \lambda_{2} \\
\lambda_{1} & -\lambda_{2}
\end{array}\right)
$$

Other values of parameters remain unchanged. The estimated parameters for different values of $\left(\lambda_{1}, \lambda_{2}\right)$ and $n$ are presented in Tables 22.2 and 22.3. The difference between the intensity parameters, $\lambda_{1}$ and $\lambda_{2}$ is apparently noticeable on the estimated parameters. When the difference is low, the estimated results closely agree to the actual values for all degrees of moments. In cases where the differences between the intensity parameters are quite substantial, for example $\lambda_{1}=0.25, \lambda_{2}=5$, the estimated results are inaccurate for low degree of moments. However, by using a higher degree of moment, e.g., $n=4$, we still obtain the results closer to actual parameters.

After we estimate the unknown parameters, we calculate the call option prices in each states by solving Eq. (22.6) and Eq. (22.7). Figure 22.1 shows the estimated option prices and the actual values using $\lambda_{1}=0.25, \lambda_{2}=2$ and $n=2$. Note that the computed values agree very well with the actual data.
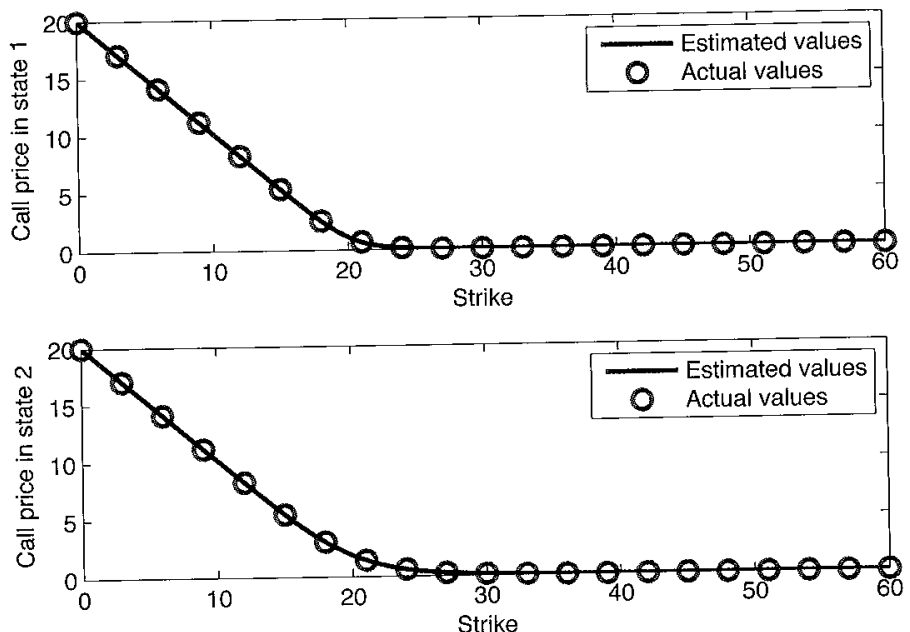

Fig. 22.1. Actual and estimated call prices: $\lambda_{1}=0.25, \lambda_{2}=2$ and $n=2$ 
Table 22.2. Example 2: Estimated parameters for different $\lambda$ and $n$ with $\sigma_{1}=0.1$ and $\sigma_{2}=0.3$

\begin{tabular}{|c|c|c|}
\hline$\left(\lambda_{1}, \lambda_{2}\right)$ & $\begin{array}{l}\text { Estimated } \\
\text { parameter }\end{array}$ & $\frac{n}{2}$ \\
\hline$(0.25,0.5)$ & $\begin{array}{c}\left(\lambda_{1}, \lambda_{2}\right) \\
\left(\sigma_{1}, \sigma_{2}\right) \\
\left(\mathrm{rmse}_{1}, \mathrm{rmse}_{2}\right)\end{array}$ & $\begin{array}{l}(0.2578,0.4708) \\
(0.0976,0.3001) \\
(0.0022,0.0051)\end{array}$ \\
\hline$(0.25,1)$ & $\begin{array}{c}\left(\lambda_{1}, \lambda_{2}\right) \\
\left(\sigma_{1}, \sigma_{2}\right) \\
\left(\mathrm{rmse}_{1}, \mathrm{rmse}_{2}\right)\end{array}$ & $\begin{array}{l}(0.2604,0.9602) \\
(0.0974,0.2997) \\
(0.0024,0.0039)\end{array}$ \\
\hline$(0.25,2)$ & $\begin{array}{c}\left(\lambda_{1}, \lambda_{2}\right) \\
\left(\sigma_{1}, \sigma_{2}\right) \\
\left(\text { rmse }_{1}, \text { rmse }_{2}\right)\end{array}$ & $\begin{array}{l}(0.2672,1.9039) \\
(0.0970,0.2979) \\
(0.0027,0.0026)\end{array}$ \\
\hline$(0.25,5)$ & $\begin{array}{c}\left(\lambda_{1}, \lambda_{2}\right) \\
\left(\sigma_{1}, \sigma_{2}\right) \\
\left(\text { rmse }_{1}, \mathrm{rmse}_{2}\right)\end{array}$ & $\begin{array}{l}(0.3044,1.8150) \\
(0.0954,0.2253) \\
(0.0047,0.0145)\end{array}$ \\
\hline$(1,0.5)$ & $\begin{array}{c}\left(\lambda_{1}, \lambda_{2}\right) \\
\left(\sigma_{1}, \sigma_{2}\right) \\
\left(\text { rmse }_{1}, \text { rmse }_{2}\right)\end{array}$ & $\begin{array}{l}(1.0059,0.4630) \\
(0.0983,0.3001) \\
(0.0010,0.0053)\end{array}$ \\
\hline$(1,2)$ & $\begin{array}{c}\left(\lambda_{1}, \lambda_{2}\right) \\
\left(\sigma_{1}, \sigma_{2}\right) \\
\left(\text { rmse }_{1}, \mathrm{rmse}_{2}\right)\end{array}$ & $\begin{array}{c}(1.0159,1.9301) \\
(0.0977,0.2993) \\
\left(7.96 \times 10^{-4}, 0.0029\right)\end{array}$ \\
\hline$(1,5)$ & $\begin{array}{c}\left(\lambda_{1}, \lambda_{2}\right) \\
\left(\sigma_{1}, \sigma_{2}\right) \\
\left(\mathrm{rmse}_{1}, \mathrm{rmse}_{2}\right)\end{array}$ & $\begin{array}{l}(1.0518,4.3958) \\
(0.0962,0.2887) \\
(0.0015,0.0017)\end{array}$ \\
\hline$(1,8)$ & $\begin{array}{c}\left(\lambda_{1}, \lambda_{2}\right) \\
\left(\sigma_{1}, \sigma_{2}\right) \\
\left(\mathrm{rmse}_{1}, \mathrm{rmse}_{2}\right)\end{array}$ & $\begin{array}{l}(1.1127,2.0694) \\
(0.0946,0.2077) \\
(0.0044,0.0082)\end{array}$ \\
\hline$(2,5)$ & $\begin{array}{c}\left(\lambda_{1}, \lambda_{2}\right) \\
\left(\sigma_{1}, \sigma_{2}\right) \\
\left(\mathrm{rmse}_{1}, \mathrm{rmse}_{2}\right)\end{array}$ & $\begin{array}{c}(2.0416,4.8157) \\
(0.0974,0.2980) \\
\left(5.11 \times 10^{-4}, 0.0014\right)\end{array}$ \\
\hline$(2,8)$ & $\begin{array}{c}\left(\lambda_{1}, \lambda_{2}\right) \\
\left(\sigma_{1}, \sigma_{2}\right) \\
\left(\mathrm{rmse}_{1}, \mathrm{rmse}_{2}\right)\end{array}$ & $\begin{array}{c}(2.0934,7.4342) \\
(0.0961,0.2937) \\
\left(6.35 \times 10^{-4}, 4.58 \times 10^{-4}\right)\end{array}$ \\
\hline
\end{tabular}

Table 22.3. Example 2 (continued): Estimated parameters for different $\lambda$ and $n$ with $\sigma_{1}=0.1$ and $\sigma_{2}=0.3$

\begin{tabular}{|c|c|c|c|}
\hline \multirow[b]{2}{*}{$\left(\lambda_{1}, \lambda_{2}\right)$} & \multirow{2}{*}{$\begin{array}{l}\text { Estimated } \\
\text { parameter } \\
\end{array}$} & \multicolumn{2}{|l|}{$n$} \\
\hline & & 3 & 4 \\
\hline$(0.25,0.5)$ & $\begin{array}{c}\left(\lambda_{1}, \lambda_{2}\right) \\
\left(\sigma_{1}, \sigma_{2}\right) \\
\left(\mathrm{rmse}_{1}, \mathrm{rmse}_{2}\right)\end{array}$ & $\begin{array}{l}(0.2529,0.4474) \\
(0.0987,0.2990) \\
(0.0014,0.0060)\end{array}$ & $\begin{array}{c}(0.2500,0.3956) \\
(0.0993,0.2965) \\
\left(8.21 \times 10^{-4}, 0.0084\right)\end{array}$ \\
\hline$(0.25,1)$ & $\begin{array}{c}\left(\lambda_{1}, \lambda_{2}\right) \\
\left(\sigma_{1}, \sigma_{2}\right) \\
\left(\text { rmse }_{1}, \text { rmse }_{2}\right)\end{array}$ & $\begin{array}{l}(0.2541,0.9414) \\
(0.0985,0.2989) \\
(0.0015,0.0046)\end{array}$ & $\begin{array}{c}(0.2506,0.8910) \\
(0.0993,0.2968) \\
\left(8.94 \times 10^{-4}, 0.0064\right)\end{array}$ \\
\hline$(0.25,2)$ & $\begin{array}{c}\left(\lambda_{1}, \lambda_{2}\right) \\
\left(\sigma_{1}, \sigma_{2}\right) \\
\left(\text { rmse }_{1}, \text { rmse }_{2}\right)\end{array}$ & $\begin{array}{l}(0.2676,1.9134) \\
(0.0983,0.2982) \\
(0.0017,0.0028)\end{array}$ & $\begin{array}{l}(0.2521,1.8780) \\
(0.0091,0.2970) \\
(0.0010,0.0037)\end{array}$ \\
\hline$(0.25,5)$ & $\begin{array}{c}\left(\lambda_{1}, \lambda_{2}\right) \\
\left(\sigma_{1}, \sigma_{2}\right) \\
\left(\text { rmse }_{1}, \text { rmse }_{2}\right)\end{array}$ & $\begin{array}{l}(0.2772,3.5618) \\
(0.0974,0.2693) \\
(0.0024,0.0053)\end{array}$ & $\begin{array}{l}(0.2612,4.3568) \\
(0.0987,0.2870) \\
(0.0014,0.0021)\end{array}$ \\
\hline$(1,0.5)$ & $\begin{array}{c}\left(\lambda_{1}, \lambda_{2}\right) \\
\left(\sigma_{1}, \sigma_{2}\right) \\
\left.\text { (rmse }_{1}, \text { rmse }_{2}\right)\end{array}$ & $\begin{array}{c}(0.9982,0.4296) \\
(0.0996,0.2987) \\
\left(8.89 \times 10^{-4}, 0.0063\right)\end{array}$ & $\begin{array}{l}(0.9934,0.3593) \\
(0.1004,0.2960) \\
(0.0011,0.0085)\end{array}$ \\
\hline$(1,2)$ & $\begin{array}{c}\left(\lambda_{1}, \lambda_{2}\right) \\
\left(\sigma_{1}, \sigma_{2}\right) \\
\left(\text { rmse }_{1}, \mathrm{rmse}_{2}\right)\end{array}$ & $\begin{array}{c}(1.0028,1.9159) \\
(0.0991,0.2989) \\
\left(4.447 \times 10^{-4}, 0.0033\right)\end{array}$ & $\begin{array}{c}(0.9951,1.8564) \\
(0.1000,0.2971) \\
\left(5.71 \times 10^{-4}, 0.0042\right)\end{array}$ \\
\hline$(1,5)$ & $\begin{array}{c}\left(\lambda_{1}, \lambda_{2}\right) \\
\left(\sigma_{1}, \sigma_{2}\right) \\
\left(\mathrm{rmse}_{1}, \mathrm{rmse}_{2}\right)\end{array}$ & $\begin{array}{c}(1.0194,4.6501) \\
(0.0983,0.2984) \\
\left(7.26 \times 10^{-4}, 0.0013\right)\end{array}$ & $\begin{array}{c}(1.0007,4.8092) \\
(0.0996,0.2973) \\
\left(1.84 \times 10^{-4}, 0.0011\right)\end{array}$ \\
\hline$(1,8)$ & $\begin{array}{c}\left(\lambda_{1}, \lambda_{2}\right) \\
\left(\sigma_{1}, \sigma_{2}\right) \\
\left(\mathrm{rmse}_{1}, \mathrm{rmse}_{2}\right)\end{array}$ & $\begin{array}{l}(1.0491,5.1333) \\
(0.0974,0.2598) \\
(0.0014,0.0026)\end{array}$ & $\begin{array}{c}(1.0115,6.8723) \\
(0.0992,0.2851) \\
\left(5.94 \times 10^{-4}, 8.18 \times 10^{-4}\right)\end{array}$ \\
\hline$(2,5)$ & $\begin{array}{c}\left(\lambda_{1}, \lambda_{2}\right) \\
\left(\sigma_{1}, \sigma_{2}\right) \\
\left(\mathrm{rmse}_{1}, \mathrm{rmse}_{2}\right)\end{array}$ & $\begin{array}{c}(2.0013,4.8841) \\
(0.0996,0.2991) \\
\left(6.81 \times 10^{-4}, 0.0015\right)\end{array}$ & $\begin{array}{c}(1.9788,4.9018) \\
(0.1009,0.2994) \\
\left(9.45 \times 10^{-4}, 0.0017\right)\end{array}$ \\
\hline$(2,8)$ & $\begin{array}{c}\left(\lambda_{1}, \lambda_{2}\right) \\
\left(\sigma_{1}, \sigma_{2}\right) \\
\left(\mathrm{rmse}_{1}, \mathrm{rmse}_{2}\right)\end{array}$ & $\begin{array}{c}(2.0207,7.7349) \\
(0.0990,0.2974) \\
\left(1.62 \times 10^{-4}, 5.15 \times 10^{-4}\right)\end{array}$ & $\begin{array}{c}(1.9793,8.0197) \\
(0.1006,0.3008) \\
\left(3.91 \times 10^{-4}, 6.29 \times 10^{-4}\right)\end{array}$ \\
\hline
\end{tabular}




\subsection{Conclusion}

In this article, we developed a methodology based on the inverse Stielties moment technique to recover the parameters of a regime-switching model from option prices. In particular, the volatility of the asset price, which is the underlying variable of the option, switches over time and modulated by a continuous-time, finite-state Markov chain. The coupled system of Dupire-type PDEs was derived from the well-known coupled system of Black-Scholes PDEs. The inverse Stieltjes moment approach was adopted to formulate the PDEs forming a linear system of equations for the volatilities and the intensity parameters. We demonstrated how to apply this method to "theoretical data", which were obtained by solving the Dupire PDEs. Numerical results were presented to illustrate the accuracy of our method. We also performed various analyses for both cases when the intensity parameters of the intensity matrix $\mathbf{A}$ and the degree of the moment $n$ is varied. Our findings based on the numerical experiments on the two types of data sets indicate the following: (1) for a single intensity rate $\lambda$, the higher the intensity rate the lower accuracy of the method, and (2) for two different intensity parameters $\lambda_{1}$ and $\lambda_{1}$, the greater the difference between these intensity rates the less accurate the estimation.

\section{References}

[1] B. Dupire, Pricing with a smile, Risk. 7(1), 18-20, (1994).

[2] P. P. Boyle and D. Thangaraj, Volatility estimation from observed option prices, Decisions in Economics and Finance. 23(1), 31-52, (2000).

[3] L. B. G. Andersen and R. Brotherton-Ratcliffe, The equity option volatility smile: An implicit finite-difference approach, Journal of Computational Finance. 1(2), 5-37, (1998).

4] M. R. Rodrigo and R. S. Mamon, A new representation of the local volatility surface, International Journal of Theoretical and Applied Finance. 11(07), 691-703, (2008).

[5] M. R. Rodrigo and R. S. Mamon, Recovery of time-dependent parameters of a Black-Scholes-type equation: An inverse Stieltjes moment approach, Journal of Applied Mathematics. 2007, (2007). Article ID 62098.

[6] I. Bouchouev and V. Isakov, The inverse problem of option pricing, Inverse Problems. 13(5), L11, (1997)

[7] Z.-C. Deng, J.-N. Yu, and L. Yang, An inverse problem of determining the implied volatility in option pricing, Journal of Mathematical Analysis and Applications. 340(1), $16-31$, (2008).

[8] C.-S. J. Chu, G. J. Santoni, and T. Liu, Stock market volatility and regime shifts in returns, Information Sciences. 94, 179-190 (October, 1996)
[9] C. M. Turner, R. Startz, and C. R. Nelson, A Markov model of heteroskedasticity, risk, and learning in the stock market, Journal of Financial Eco nomics. 25(1), 3 - 22, (1989).

[10] C. Engel and J. D. Hamilton, Long swings in the dollar: Are they in the data and do markets know it?, American Economic Review. 80(4), 689-713, (1990).

[11] G. Bekaert and R. J. Hodrick, On biases in the measurement of foreign exchange risk premiums, Journal of International Money and Finance. 12 (2), $115-138,(1993)$.

[12] C. Engel and C. S. Hakkio, The distribution of exchange rates in the EMS, International Journal of Finance \&g Economics. 1(1), 55-67, (1996).

13] M. Dahlquist and S. F. Gray, Regime-switching and interest rates in the European monetary system, Journal of International Economics. 50(2), 399-419, (2000).

[14] A. Ang and G. Bekaert, Regime switches in interest rates, Journal of Business \&f Economic Statistics. 20(2), 163-82, (2002).

[15] R. J. Elliott and R. S. Mamon, An interest rate model with a Markovian mean reverting level, Quantitative Finance. 2, 454-458(5), (2002).

[16] R. J. Elliott and P. E. Kopp, Mathematics of Financial Markets. (Springer, Berlin Heidelberg New York, 1999).

[17] R. J. Elliott, L. Chan, and T. K. Siu, Option pricing and Esscher transform under regime switching, Annals of Finance. 1(4), 423-432, (2005).

[18] W. Ching, K. Siu, and L. Li, Pricing exotic options under a higher-order hidden Markov model, Journal of Applied Mathematics and Decision Sciences. pp. 1-15, (2007)

[19] R. S. Mamon and M. R. Rodrigo, Explicit solutions to European options in a regime-switching economy, Operations Research Letters. 33(6), 581-586, (2005).

[20] P. Boyle and T. Draviam, Pricing exotic options under regime switching, Insurance: Mathematics and Economics. 40(2), 267 - 282, (2007).

[21] J.-C. Duan, I. Popova, and P. Ritchken, Option pricing under regime switching, Quantitative Finance. 2, 116-132, (2002).

[22] T. K. Siu, C. Erlwein, and R. S. Mamon, The pricing of credit default swaps under a Markov-modulated Merton's structural model, North American Actuarial Journal. 12(1), 229-238, (2008).

[23] R. J. Elliott and J. van der Hoek, An application of hidden Markov models to asset allocation problems, Finance and Stochastics. 1, 229-238, (1997).

[24] R. J. Elliott, L. Aggoun, and J. B. Moore, Hidden Markov Models: Estimation and Control. (Springer, Berlin, 1994).

[25] X. Guo, Information and option pricings, Quantitative Finance. 1, 38-44, (2001). 